\title{
Improving Decision Making under Uncertainty Conditions in Highly Dynamical Contexts
}

\author{
Roberto G. Aldunate, Cesar Navarro, Daniel San Martin \\ Center for Innovation and Applied Research
}

\begin{abstract}
Decision making during response to large scale disasters requires to be conducted as efficient and effective as possible in order to mitigate the multiple-domain impact of those disasters in our communities. Incomplete information and cascading effects, i.e.; one person's decisions affecting the same person or others at a future moment, limits the performance of decision makers in this context. This article presents a research effort that involves the development of a video game-based web platform, which includes wildfire, flooding, and earthquake scenarios, that in conjunction with an expert playing a facilitator role, aimed at assessing and improving trainees' decision making. The results obtained are two-fold. First, they highlight that trainees improve their decision making performance quickly using a video game based simulator with the support of an expert facilitating knowledge acquisition during debrief postsimulation sessions. Second, experts and trainees have signature decision making performance curves. The main conclusions from this research effort are that the video gamebased simulator is perceived as a valid tool to support decision making training, and that this technology improves decision making conducted under incomplete information and cascading effects.
\end{abstract}

Keywords: cascading effects; decision making; incomplete information; simulator; video game 